\title{
Utilização da Realidade Virtual na Indução de Estados Afetivos
}

\section{The Impact of Virtual Reality on the Induction of Affective States}

\author{
C.M. Teixeira, A.S. Gonçalves, C.C. Gonçalves, C.P. Martins, L.M. Matos
}

ARTIGO ORIGINAL

ORIGINAL ARTICLE

\begin{abstract}
RESUMO
O objetivo principal deste estudo foi perceber qual o impacto de dois ambientes virtuais na indução de estados afetivos sentimento de presença e se esses diferiam em função do sexo e nível de experiência. Os objetivos específicos passam por comparar os diferentes níveis de experiência de jogo em função das dimensões de presença, comparar a ausência e a presença de realidade virtual na indução de estados afetivos e comparar os níveis de regularidade na utilização de jogos de vídeo e o seu impacto nos estados afetivos. A amostra consistiu em 20 participantes com idades compreendidas entre os 18 e os 44 anos de ambos os sexos. Todos os selecionados frequentavam o ensino superior no norte de Portugal. A amostra foi dividida em dois grupos, 10 para cada ambiente virtual onde um grupo realizou a experiência com o jogo Aboots Demo e o outro realizou com o jogo Resident Evil-7. Foi utlizado um questionário Sociodemográfico, o Perfil de Estados Afetivos e o IPQp para a recolha de dados. Os resultados evidenciaram não existir qualquer efeito ao nível dos domínios da presença. Em conclusão, quanto aos estados afetivos, a Tensão, Fadiga e Vigor são os mais significativos.
\end{abstract}

Palavras-chave: realidade virtual, estados afetivos, regularidade, presença, experiência

\begin{abstract}
The main objective of this study is to understand if two different virtual environments induce different affective states in the participants. The specific goals are to compare the different levels of game experience according to the dimensions of presence, compare the absence and presence of virtual reality in the induction of affective states, and compare the levels of regularity in the use of video games and its impact on affective states. For this purpose, 20 participants were collected for convenience, from a Portuguese University in the north of Portugal, aged between 18 and 44 years of both sexes, which were distributed 10 for each virtual environment where a group performed the experience with the game Aboots Demo and the other group performed with the game Resident Evil 7. A Sociodemographic Questionnaire, the Affective States Profile, and the IPQp were used for data collection. The main conclusions of this study showed different affective states in the different virtual reality conditions/environments, where Tension, Vigor, and Fatigue are the most relevant.

Keywords: virtual reality, affective states, regularity, presence, competence
\end{abstract}

Submetido: 07. 17.2017 | Aceite: 03.24.2018

Carla M. Teixeira. Dept. Educação e Psicologia, Universidade de Trás-os-Montes e Alto Douro, Vila Real, Portugal.

Adriana C. S. Gonçalves, Catarina S. C. Gonçalves, Cátia D. P. Martins, Luís D. M. Matos. Universidade de Trás-os-Montes e Alto Douro, Vila Real, Portugal.

Endereço para correspondência: Carla M. Teixeira. Quinta de Prados, Apt. 1013, ECHS-1, Gab. 2.27, 5000-881 Vila Real, Portugal.E-mail: cteixeir@utad.pt 
O futuro da Psicologia, assim como dos avanços na área das tecnologias, passa pelo aprofundamento do conhecimento sobre como os fatores humanos influenciam o desenvolvimento e a consequente oferta de novos produtos tecnológicos, assim como na utilização destes equipamentos na prestação de diversos serviços, assim como nas intervenções terapêuticas (APA, 2017). Um exemplo destes avanços é a utilização da realidade virtual no tratamento de diversas perturbações como a perturbação de pânico (Carvalho, Freire, \& Nardi, 2008), a perturbação de ansiedade (Parsons \& Rizzo, 2008), várias fobias (Parsons, \& Rizzo, 2008; Ribeiro \& Monteiro, 2015), perturbações alimentares (Botella, et al., 2007) e perturbações do espetro de autismo (Ribeiro \& Monteiro, 2015; Strickland, 1997). Esta tecnologia está, ainda, a ser utilizada na recuperação cognitiva de doentes com traumatismo crânio-encefálico (Ribeiro \& Monteiro, 2015) e na tentativa de conscientizar pacientes com esquizofrenia, relativamente às alucinações de que sofrem (Costa \& Carvalho, 2003).

A realidade virtual é descrita por vários autores como uma interface avançada do usuário com o computador onde o mesmo não fica apenas em frente ao monitor, mas sim imerso num mundo artificial e tridimensional. É uma interface que simula um mundo real que permite ao usuário interagir como se o mundo virtual fosse efetivamente real, enquanto está a ser utilizado (Ribeiro \& Zorzal, 2011). Quando imerso no mundo virtual, o usuário tem uma pequena ou até nenhuma consciência de que está a usar uma interface usuário-computador (Netto, Machado, \& Oliveira, 2002; Costa \& Cravalho, 2003). No fundo, a RV é um espelho da realidade física.

A RV tem como pilares três ideias fundamentais: a imersão, que diz respeito ao sentimento de fazer parte do ambiente; a interação que está ligada à capacidade do computador para detetar os movimentos do usuário e modificar de forma instantânea o mundo virtual, consoante as ações efetuadas sobre ele (capacidade reativa); e o envolvimento que está ligado ao grau de motivação para o envolvimento de uma pessoa para uma dada atividade, o qual se obtém através dos estímulos dos sentidos humanos (audição, visão e tato) (Costa \& Carvalho, 2003; Netto, et al., 2002;). Neste caso, existem vários dispositivos, capacete de visualização (Head-mounted displays - HMD), dispositivos de rastreio, luvas eletrónicas, joysticks, que permitem ao usuário navegar através de um ambiente virtual e interagir com objetos virtuais.

Costa e Carvalho (2003) e Ribeiro e Monteiro (2015) afirmam que ambientes virtuais criam relações afetivas e são eficazes na indução de respostas emocionais. Estudos realizados mostraram que determinadas variações fisiológicas (como a frequência cardíaca e a condutividade da pele) estão associados a diferentes estados afetivos em experiências onde os participantes estavam envolvidos numa realidade virtual (Monteiro, 2011; Ribeiro \& Monteiro, 2015; Tijiboy, Pereira, \& Woiciechoski, 2009; Yannakakis, Martinez, \& Garbarino, 2016). Isto mostra que a tecnologia RV tem vindo a permitir o desenvolvimento de estímulos com propriedades muito próximas do real, o que confere uma maior sensação de presença no mundo virtual (Ribeiro \& Monteiro, 2015). Os estados afetivos são, no fundo estados que revelam as emoções, sentimentos, tendências e paixões e que variam numa tonalidade que vai do agradável ao desagradável (Kahneman, 1999; Jaquet, 2013; Longhi, Bercht, \& Behar, 2007; Tijiboy, et al., 2009).

As emoções interferem no modo como as pessoas atribuem significados às suas experiências e até que ponto elas se podem sentir presentes num ambiente manipulado (Riva et al., 2007). Ribeiro e Monteiro (2015) quiseram perceber se cenários de indução afetivas em 2D e 3D mostravam níveis de sensação de presença diferentes. Várias são as investigações que estudam esta relação 
entre estados afetivos e realidade virtual e, por isso, sabendo que cenários de indução afetiva 2D e 3D mostram diferentes sensações de presença dos jogadores (Ribeiro \& Monteiro, 2015), importa perceber se estes dois cenários de realidade virtual (variável independente) provocam estados afetivos (variável dependente) diferentes nos participantes.

Estudos mostram que a dimensão lúdica dos jogos facilita o interesse e a motivação pela sua utilização, ou seja, a regularidade da utilização dos mesmos. A literatura da especialidade sugere que os níveis de regularidade com que se joga estão intrinsecamente associados aos níveis de desafio apresentado, a curiosidade do jogador, assim como a fantasia representada no próprio jogo. Estes elementos são definidos como fatores motivacionais, uma vez que estimulam a curiosidade e o desejo para se ganhar competência que, por sua vez, funcionam como um desafio à inteligência e que leva a uma maior retenção de informação nos participantes (Arriaga, Silva, \& Esteves, 2001; Dorval \& Pervin, 1986; Okagaki \& Frensch, 1994). As mesmas fontes realçam que os jogos de vídeo fazem parte do quotidiano dos jovens e afirmam que estes usam esta prática como estratégia de relaxamento, distração, e pelo prazer que sentem com o desafio para superarem as etapas/ níveis do jogo. Em acréscimo, a componente de socialização promove a sensação de prazer quando o fazem com amigos. Por outras palavras, a regularidade com que se joga pode ser predita por diversos fatores. Os resultados sugerem ainda que os jogos são mais populares entre o sexo masculino do que entre o sexo feminino.

A regularidade de jogo é fomentada por jogos online pois estes permitem a formação de laços sociais, promovendo o reforço social, e apresentam propriedades imersivas onde os jogadores experienciam um ambiente alternativo sentindo uma necessidade de progredir e superar etapas (Batista, Quintão, \& Barroso, 2008; Suzuki, Matias, Silva, \& Oliveira, 2009).
A regularidade e familiaridade que os utilizadores de jogos de vídeo apresentam com os equipamentos de realidade virtual, repercutem-se na maior facilidade de quem joga na manipulação de qualquer tipo de equipamento que pode servir funções de controlador, uma vez que os jovens desde novos se habituam à utilização de teclados, computadores, telemóveis, comandos e joysticks. Para além desta vantagem prática, importa salientar que os indivíduos habituados a jogar, quando expostos a contextos em que estão a ocorrer vários acontecimentos em simultâneo, apresentam maiores capacidades para identificar e de se concentrar naquilo que é mais importante para a tarefa em causa, uma vez que estão mais treinados a filtrar e selecionar de entre toda a informação disponibilizada aquela que é a mais relevante para a ação necessária (Green \& Bavelier, 2006; Lopes \& Oliveira, 2012).

De acordo com a descrição de Lopes e Oliveira (2012) o Instituto Nacional de Estatística (INE) afirma que em Portugal 91,5\% dos adolescentes afirmaram já alguma vez ter jogado videojogos e que $79 \%$ das crianças e jovens portugueses, entre os dez e os quinze anos, utilizaram a internet para jogar ou descarregar jogos, imagens, filmes ou músicas. Apesar de existir um discurso infundadamente negativo relativamente aos jogos, as evidências empíricas sugerem que a exposição a jogos de vídeo é um elemento que oferece o potencial de ser utilizado em processo de treino que visem o desenvolvimento de competências motoras e cognitivas, nomeadamente aquelas que se relacionam como as competências visuais, espaciais, e de memória, capacidades estas que são de extrema importância no progresso de muitas profissões (Lopes \& Oliveira, 2012; Boot, Kramer, Simons, Fabiani, \& Gratton, 2008).

A regularidade com que os indivíduos jogam pode ser influenciada e estimulada por diversos fatores. Assim, como o presente estudo pretendemos perceber se a regularidade de jogo (variável independente) consegue influenciar os estados afetivos dos utilizadores (variável 
dependente). Quando realizámos a revisão da literatura, não encontramos estudos que já tivessem estudado a relação destas variáveis e, mais uma vez, constatámos a crescente relevância da investigação que visa o aprofundamento do conhecimento nesta área dos fatores humanos associados à utilização de tecnologias.

No desenvolvimento dos ambientes virtuais há a preocupação de assegurar uma qualidade de imagem que permita a maior imersão possível. Essa noção de envolvimento tem por base a sensação de presença vivenciada pelo utilizador. A sensação de presença diz respeito à ideia de que um ambiente virtual é tão mais real quando as respostas das pessoas a estímulos virtuais são proporcionais às respostas das pessoas a estímulos reais (Slater, Lotto, Arnold, \& Sanchez-Vives, 2009; Anderson \& Price 2007; Ribeiro \& Monteiro, 2015). A presença pode ser considerada como um estado subjetivo, avaliado por meio de questionários de autorrelato, dependentes somente da memória do participante, decorrente da experiência. No entanto, nos últimos tempos o objetivo tem sido realizar uma avaliação contínua da experiência e, para isso, foram introduzidas medidas mais objetivas, nomeadamente as medidas fisiológicas periféricas. As mais utilizadas são o Ritmo Cardíaco e a Atividade Eletrodérmica, pois foi comprovada a existência de uma relação diretamente proporcional entre a desaceleração cardíaca e os elevados níveis de presença (Ribeiro \& Monteiro, 2015).

A definição de sensação de presença aplica-se tanto para ambientes de realidade mista como para realidade virtual pura. Esta definição inclui, automaticamente, a interação, a capacidade de cada um para interagir com o ambiente e, especialmente, a capacidade do sujeito para usar o corpo inteiro na interação quando apropriado (Slater, et al., 2009; Baños, et al., 2004; Carvalho, et al., 2008; MacDonald, 2006).

Investigações anteriores têm vindo a mostrar que a sensação de presença é algo muito estudado pela comunidade científica e como tal já várias conclusões se tiraram e outras ainda se mantêm em discussão (IJsselsteijn \& Riva, 2003). Um estudo realizado por Baños, et al. (2004) mostraram que a sensação de presença é afetada por ambientes afetivos e, uma outra investigação realizada por Ribeiro e Monteiro (2015) avançou com a ideia que a sensação de presença é também afetada por ambientes virtuais 2D e 3D.

$\mathrm{Na}$ pesquisa bibliográfica realizada, encontrámos nos estudos já referidos a argumentação que há uma relação entre a perceção de presença de jogo e os estados afetivos com a experiência do jogador. No entanto, a demonstração destas relações carecem de evidências empíricas. O presente estudo visa dar um contributo no preenchimento dessa lacuna.

Face ao atual estado da arte, no presente estudo, questionamos se serão diferentes estados afetivos vivenciados em diferentes cenários, como por exemplo os $2 \mathrm{D}$ e $3 \mathrm{D}$. Assim, o objetivo geral da presente investigação é perceber se existem diferenças entre as perceções experienciadas quando expostos a vivências em realidade virtual e em realidade não virtual na indução de estados afetivos. Os objetivos específicos são comparar por sexo, experiência num jogo com exposição aos equipamentos de realidade virtual e regularidade com que joga na perceção de presença e na indução de estados afetivos.

\section{MÉTODO}

O presente estudo é de natureza transversal, comparativo e recorre a um desenho pré-pós-teste. Como variáveis independentes temos o sexo a regularidade de jogo, a experiência de jogo e as duas condições/ambientes de realidade virtual. As variáveis dependentes foram os estados afetivos e a perceção de presença.

\section{Amostra}

Participaram neste estudo 20 indivíduos recolhidos por conveniência, de uma Universidade portuguesa do norte, com idades 
compreendidas entre os 18 e os 44 anos e de ambos os sexos (11 do sexo feminino, 9 do sexo masculino). Destes, todos tinham nacionalidade portuguesa, com o ensino secundário concluído. $95 \%$ são ainda estudantes. 55\% $(\mathrm{N}=11)$ dos sujeitos consideram-se como não jogadores, $35 \%(\mathrm{~N}=7)$ jogam entre 1 a 3 vezes por semana e $10 \%(\mathrm{~N}=2)$ entre 4 e 7 vezes por semana. Por sua vez, 13 (65\%) participantes afirmam que jogam 0 horas por dia, 5 (25\%) jogam entre 1 e 2 horas por dia, e 2 (10\%) jogam entre 3 a 4 horas por dia. $60 \%(\mathrm{~N}=12)$ consideram-se pouco experientes e $40 \%(\mathrm{~N}=8)$ consideram-se experientes no que confere às suas competências de jogo.

O grupo de 20 foi subdividido, aleatoriamente, em dois grupos de 10, um dos grupos realizou a experiência sem que para o efeito tivesse visionado as imagens num sistema de realidade virtual. $\mathrm{O}$ segundo grupo foi aquele que fez o visionamento recorrendo ao equipamento da realidade virtual.

\section{Instrumentos}

Para a recolha de dados optámos por utilizar um questionário Sociodemográfico e de onde foi possível retirar as variáveis independentes como sexo, idade, habilitações literárias, regularidade com que joga e experiência no tipo de jogo (2D e 3D). Foi utilizada a escala de Perfil de Humor (POMS) (Viana, Almeida, \& Santos 2001), para avaliar os estados afetivos dos participantes. A aplicação foi feita após a realização da experiência em ambos os grupos (contacto ou não com os equipamentos de realidade virtual).

O POMS consiste em seis escalas: Tensão, Depressão, Hostilidade, Vigor, Fadiga e Confusão. Utilizámos a versão reduzida com 6 itens cada sub-escala, o que faz um total de 36 itens que são apresentados numa escala de Likert de 5 pontos $(0=$ Nunca; $1=$ Um pouco; 2 = Moderadamente; 3 = Bastante; 4 = Muitíssimo). Os itens são cotados na mesma direção, com a exceção dos seguintes: um item na escala de Tensão (Tranquilo) e dois itens na escala de
Confusão (Eficaz e Competente). Nestes casos, a resposta ao item deve ser invertida antes de adicionar aos restantes. Esta escala foi validade e adaptada para a população portuguesa por Viana, et al. (2001). No que diz respeito à sua fidelidade, na versão portuguesa todas as escalas apresentam coeficientes acima de 0.7 , o que atesta a boa consistência interna das suas escalas. Portanto, a versão reduzida do POMS revela-se um instrumento válido e preciso para medir os estados de humor.

Para medir a sensação/ dimensão de presença dos participantes, foi utilizado o questionário IPQp. O IPQp é um questionário de autorrelato validado e foi adaptado para a população portuguesa por Vasconcelos-Raposo et al., (2016). Este instrumento visa medir as seguintes variáveis: presença espacial, envolvimento, realidade e a presença global.

\section{Procedimentos}

Este estudo foi realizado em dois momentos distintos, devido à disponibilidade dos equipamentos, dos participantes e do espaço com condições necessárias para a sua realização. Assim, os participantes começaram a experiência com o preenchimento de um questionário sociodemográfico com o objetivo de ser feita uma recolha de dados para a realização da presente investigação e, de seguida, foi-lhes pedido que se sentassem, confortavelmente, em frente ao monitor de um computador. Posteriormente, foi solicitado aos participantes que lessem atentamente as instruções da experiência. Houve o cuidado de dar a oportunidade a cada um dos participantes se assegurarem que haviam entendido todas indicações apresentadas. Os assistentes de investigação, responsáveis pela entrega e recolha dos questionários foram treinados a dar esclarecimentos sem que nesse processo indiciassem qualquer tipo de resposta a ser dada pelos participantes. Em seguida, foi explicado aos participantes o funciomamento dos comandos que iriam utilizar e foram colocados os óculos de realidade virtual fazendo os ajustes necessários 
a cada participante. Depois de assegurar todos estes procedimentos passou-se então à realização da experiência onde um grupo de 10 participantes teve contacto com o vídeo jogo Aboots Demo com os equipamentos de realidade virtual, e um outro grupo de 10 participantes que teve contacto com outro vídeo jogo, Resident Evil 7, sem equipamentos de realidade virtual. Ambos os jogos são de natureza idêntica, facilitando o mesmo tipo de estados de humor. Num dos ambientes os participantes utilizavam os óculos de realidade virtual ao qual denominámos de "Presença da realidade virtual" e um outro ambiente onde os participantes não tinham contacto com os óculos de realidade virtual ao qual denominamos por "Ausência da realidade virtual". Finalmente, após a realização da experiência, foi pedido aos participantes que preenchessem um questionário para que fosse possível a recolha de dados sobre a experiência dos participantes.

\section{Análise Estatística}

Para testar as hipóteses do estudo recorremos à MANOVA, uma vez que se verificou a normalidade da distribuição dos dados, medida pelo Skewness e Kurtosis que apresentaram valores entre -1 e 1. Para estas análises estatísticas foi utilizado o IBM-SPSS, versão 23.0. O valor adotado para considerar diferenças significativas foi $p=.05$. Foi ainda tomada em consideração a dimensão dos efeitos estatísticos estabelecendo-se como critérios os seguintes: pequeno $>.01$, moderado $>.06$ e forte $>.14$. Cabe referir que os dados foram tratados tendo em conta um intervalo de confiança de $95 \%$.

\section{RESULTADOS}

A MANOVA foi utilizada para comparar as diferentes variáveis cujos resultados são apresentados nos seguintes quadros.

Os resultados da MANOVA com as variáveis nível de experiência $\mathrm{x}$ sexo, nas dimensões da

Quadro 1

Médias (M), Desvio Padrão (DP) por efeito da variável "Presença" na Análise Univariada.

\begin{tabular}{lccccc}
\hline \multicolumn{1}{c}{ Variáveis } & $\begin{array}{c}\text { Sexo Masculino } \\
\mathrm{M} \pm \mathrm{DP}\end{array}$ & $\begin{array}{c}\text { Sexo Feminino } \\
M \pm D P\end{array}$ & $F$ & $p$ & $\boldsymbol{\eta}_{p}{ }^{2}$ \\
\hline Presença Espacial & $3.64 \pm .34$ & $3.86 \pm .40$ & 1.174 & .296 & .073 \\
Envolvimento & $3.19 \pm .96$ & $3.15 \pm .58$ & .337 & .570 & .022 \\
Realismo Experienciado & $2.63 \pm .70$ & $2.77 \pm .71$ & .270 & .611 & .018 \\
\hline
\end{tabular}

presença sugerem que há um efeito estatístico pequeno $\left(\mathrm{F}_{(3,13)}=.155, p=.925, \eta_{p}^{2}=.034, \mathrm{PO}=\right.$ .071). Com base nos valores descritos no quadro 2 , podemos afirmar que não foram encontradas dife- renças estatisticamente significativas em todas as dimensões da Presença.

Relativamente às comparações ao nível das sensações de Presença (IPQp: Presença Espa-

Quadro 2

Médias (M), Desvio Padrão (DP) por efeito da variável "Presença" na Análise Univariada.

\begin{tabular}{lccccc}
\hline \multicolumn{1}{c}{ Variáveis } & $\begin{array}{c}\text { Pouco Experiente } \\
\mathrm{M} \pm \mathrm{DP}\end{array}$ & $\begin{array}{c}\text { Experiente } \\
M \pm D P\end{array}$ & $F$ & $p$ & $\boldsymbol{\eta}_{p}{ }^{2}$ \\
\hline Presença Espacial & $3.79 \pm .42$ & $3.72 \pm .34$ & .119 & .734 & .007 \\
Envolvimento & $3.12 \pm .64$ & $3.25 \pm .94$ & .125 & .728 & .007 \\
Realismo Experienciado & $2.72 \pm .67$ & $2.68 \pm .76$ & .016 & .900 & .001 \\
\hline
\end{tabular}


cial, Envolvimento, Realismo Experienciado) dos participantes era predita que ao nível da experiência de jogo se encontrariam diferenças entre os grupos. Os resultados indicaram que na comparação entre sexos apenas a Presença Espacial apresentou um efeito estatístico moderado. Na comparação realizada entre os dois domínios de experiência dos jogadores (experientes ou pouco experientes) os resultados mostraram um efeito estatístico pequeno em todas as escalas do IPQp. No entanto, quando da tomada de decisão sobre a relevância estatística, tando por base a articulação entre $p, \eta_{p}^{2}$ e PO, constatámos que os valores obtidos eram negligenciáveis.
A significância da análise multivariada sobre o efeito da experiência do jogador na presença $\left(\mathrm{F}_{(3,16)}=.122, p=0.946, \eta_{p}^{2}=.022, \mathrm{PO}=\right.$ $.068)$ indica que o efeito estatístico de todas as variáveis foi pequeno. A análise univariada nas três dimensões evidenciam não existir qualquer resultado com dimensão significativa nas comparações entre os grupos.

Os valores do Quadro 3 mostram que as comparações entre sexos apresentam um efeito estatístico moderado em três das variáveis estudadas: Tensão, Vigor e Fadiga em que foram as mulheres que apresentaram valores mais altos nas primeiras duas e os homens na Fadiga.

Quadro 3

Médias (M), Desvio Padrão (DP) por efeito da variável "Estados Afetivos" na Análise Univariada IC 95\%.

\begin{tabular}{lccccc}
\hline \multicolumn{1}{c}{ Variáveis } & $\begin{array}{c}\text { Sexo Masculino } \\
\text { M DP }\end{array}$ & $\begin{array}{c}\text { Sexo Feminino } \\
\text { M DP }\end{array}$ & $F$ & $p$ & $\boldsymbol{\eta}_{p}{ }^{2}$ \\
\hline Tensão & $6.44 \pm 5.15$ & $7.45 \pm 6.40$ & 1.526 & .235 & .087 \\
Depressão & $1.77 \pm 2.99$ & $1.00 \pm 2.48$ & .001 & .973 & .001 \\
Hostilidade & $2.00 \pm 4.21$ & $0.90 \pm 1.92$ & .031 & .863 & .002 \\
Vigor & $10.55 \pm 3.32$ & $12.09 \pm 4.39$ & .874 & .364 & .052 \\
Fadiga & $2.33 \pm 4.60$ & $0.54 \pm 1.50$ & 1.363 & .260 & .078 \\
Confusão & $9.66 \pm 5.19$ & $9.00 \pm 5.11$ & .045 & .834 & .003 \\
\hline
\end{tabular}

A comparação dos sujeitos por condições/ ambientes de ausência/ presença da realidade virtual (utilização ou não dos óculos de realidade virtual), segundo uma análise multivariada $\left(\mathrm{F}_{(6,11)}=.894, p=.532, \eta_{p}^{2}=.328, \mathrm{PO}=.224\right)$. A análise univariada (ver quadro 4) mostra-nos que o efeito estatístico da Tensão, da Depressão, da Hostilidade é muito forte e nas escalas do Vigor e da Fadiga é nulo.

Relativamente às comparações entre homens e mulheres, nas escalas do POMS, a análise multivariada evidenciou um efeito estatístico forte entre as condições Ausência/Presença da realidade virtual numa $\left(\mathrm{F}_{(6,13)}=1.803, p=.175, \eta_{p}^{2}=\right.$ $.454, \mathrm{PO}=.468) . \mathrm{A}$ análise univariada mostrou que há um efeito muito forte nas variáveis Tensão, Depressão e Hostilidade Vigor, um efeito pequeno na Fadiga e um efeito forte na Confusão.

A análise multivariada por sexo demonstrou um efeito estatístico forte nas comparações entre níveis de regularidade de jogo ao nível dos estados afetivos $\left(\mathrm{F}_{(6,9)}=.337, p=.901\right.$, $\eta_{p}^{2}=.183$, PO $\left.=.101\right)$.

A análise multivariada por sexo demonstrou um efeito estatístico forte nas comparações entre níveis de regularidade de jogo ao nível dos estados afetivos $\left(\mathrm{F}_{(6,9)}=.337, p=.901, \eta_{p}^{2}=.183\right.$, $\mathrm{PO}=.101)$. A análise univariada evidenciou o efeito estatístico relativamente à regularidade de jogo dos participantes numa análise multivariada $\left(\mathrm{F}_{(12,20)}=.827, p=.632, \eta_{p}^{2}=.293, \mathrm{PO}=.340\right)$ é muito forte. Notou-se um efeito muito forte no Vigor $\left(\eta_{p}^{2}=.290\right)$ e forte na Fadiga $\left(\eta_{p}^{2}=.143\right)$ e Confusão $\left(\eta_{p}^{2}=.047\right.$, e moderado em todas as restantes variáveis (Quadro 5).

\section{DISCUSSÃO}

O presente estudo visou perceber se variáveis 
Quadro 4

Médias (M), Desvio Padrão (DP) por efeito da variável "Estados Afetivos" na Análise Univariada.

\begin{tabular}{lccccc}
\hline \multicolumn{1}{c}{ Variáveis } & $\begin{array}{c}\text { Presença da RV } \\
\text { M } \pm \text { DP }\end{array}$ & $\begin{array}{c}\text { Ausência da RV } \\
\text { M } \pm \text { DP }\end{array}$ & $F$ & $p$ & $\boldsymbol{\eta}_{p}{ }^{2}$ \\
\hline Tensão & $4.10 \pm 2.33$ & $9.90 \pm 6.75$ & 6.585 & .019 & .268 \\
Depressão & $0.10 \pm 0.31$ & $2.60 \pm 3.40$ & 5.342 & .033 & .229 \\
Hostilidade & $0.00 \pm 0.00$ & $2.80 \pm 4.02$ & 4.846 & .041 & .212 \\
Vigor & $11.10 \pm 4.86$ & $11.70 \pm 2.94$ & .111 & .742 & .006 \\
Fadiga & $1.30 \pm 2.54$ & $1.40 \pm 4.08$ & .004 & .948 & .001 \\
Confusão & $7.40 \pm 2.63$ & $11.20 \pm 6.19$ & 3.185 & .091 & .150 \\
\hline
\end{tabular}

Quadro 5

Médias (M), Desvio Padrão (DP) por efeito da variável "Estados Afetivos" na Análise Univariada.

\begin{tabular}{|c|c|c|c|c|c|c|}
\hline Variáveis & $\begin{array}{l}0 \text { vezes por } \\
\text { semana } \\
M \pm D P\end{array}$ & $\begin{array}{c}1 \text { a } 3 \text { vezes por } \\
\text { semana } \\
M \pm D P\end{array}$ & $\begin{array}{c}4 \text { a } 7 \text { vezes por } \\
\text { semana } \\
M \pm D P\end{array}$ & $F$ & $p$ & $\eta_{p}^{2}$ \\
\hline Tensão & $8.00 \pm 6.08$ & $6.28 \pm 6.01$ & $4.00 \pm 2.82$ & .465 & .636 & .052 \\
\hline Depressão & $1.09 \pm 2.46$ & $2.14 \pm 3.33$ & $0.00 \pm 0.00$ & .584 & .568 & .064 \\
\hline Hostilidade & $1.09 \pm 1.92$ & $2.28 \pm 4.78$ & $0.00 \pm 0.00$ & .510 & .609 & .057 \\
\hline Vigor & $11.00 \pm 3.31$ & $10.28 \pm 3.94$ & $17.50 \pm 2.12$ & 3.469 & .055 & .290 \\
\hline Fadiga & $0.54 \pm 1.50$ & $3.00 \pm 5.09$ & $0.00 \pm 0.00$ & 1.418 & .269 & .143 \\
\hline Confusão & $9.36 \pm 4.86$ & $10.42 \pm 5.71$ & $5.00 \pm 5.02$ & .899 & .425 & .096 \\
\hline
\end{tabular}

como o sexo, utilização de equipamento de realidade virtual, e frequência com que os sujeitos jogam tinha alguma influência na indução de estados afetivos/ humor e níveis de presença.

A sensação de presença surge quando há substituição bem-sucedida de dados sensoriais reais por dados sensoriais virtualmente gerados. A substituição é "bem-sucedida" quando o participante numa realidade virtual, ou mista, forma perceções, responde e age sobre estes como se fossem reais (Slater, Lotto, Arnold, \& Sanchez-Vives, 2009). Como já foi referido, os estudos sobre a sensação de presença, têm sido levados a cabo relativamente a várias variáveis, mas ao nível da relação com os estados afetivos não nos foi possível encontrar referências que abordassem essas relações, tal como levamos acabo com o presente estudo. Posto isto, não temos a possibilidade de comparar os nossos resultados com os de outros estudos. Os nossos resultados sugerem que, de forma geral, não há efeitos estatísticos significativos da experiência de jogo na sensação de perceção de presença. Qualquer interpretação teórica dos resultados obtidos assumem um carater especulativo, graças à reduzida dimensão da amostra estudada.

Os resultados relativos ao objetivo do estudo no qual se pretendia perceber se os dois ambientes/condições de realidade virtual (utilização de óculos de realidade virtual-Presença da Realidade Virtual; não utilização de óculos de realidade virtual- Ausência de Realidade Virtual) induziam diferentes estados afetivos (POMS- Tensão, Depressão, Hostilidade, Vigor, Fadiga e Confusão) nos participantes, mostraram que o efeito estatístico relativamente ao sexo é forte, ou seja, há diferenças significativas entre o sexo masculino e feminino relativamente aos seus estados afetivos nas duas condições. Os fatores Tensão, 
Fadiga e Vigor mostraram efeitos relevantes nesta comparação entre sexos. Nas comparações realizadas entre os dois ambientes/ condições de realidade virtual, os resultados mostraram que os estados afetivos são influenciados pelas condições de Presença/ Ausência da Realidade Virtual. Os fatores Tensão, Depressão, Hostilidade e Confusão foram os mais significativos.

Estudos anteriormente realizados mostraram que ambientes de realidade virtual são capazes de induzir estados afetivos ou respostas emocionais aos utilizadores (Costa \& Carvalho, 2003; Ribeiro \& Monteiro, 2015). Nesta linha, os resultados indicaram que ambientes virtuais conseguem produzir estados afetivos nos participantes, neste caso, notaram-se diferenças nos estados afetivos dos participantes entre as duas condições estudadas.

Um outro objetivo pretendia compreender se a regularidade com que os participantes jogam videojogos influencia os seus estados afetivos, ou seja, comparar por nível de regularidade ( 0 vezes por semana, 1 a 3 vezes por semana, 4 a 7 vezes por semana) os estados afetivos. Os resultados apontaram que, a nível das diferenças entre sexos, se obteve um efeito estatístico forte, ou seja, que há diferenças significativas entre o sexo masculino e o sexo feminino no que respeita aos seus estados afetivos nos diferentes níveis de regularidade. O fator Vigor mostrou um efeito forte nesta comparação. Estudos realizados anteriormente indicam que há uma diferença entre os sexos na regularidade com que utilizam jogos de vídeo e argumentam que esta regularidade é maior no sexo masculino do que no sexo feminino (Suzuki, et al., 2009; Batista, et al., 2008). Os nossos resultados apontam que existem diferenças entre homens e mulheres ao nível dos estados afetivos e que estas dependem da regularidade de jogo. Na nossa amostra constatou-se que são os indivíduos do sexo masculino os que apresentam maior regularidade de jogo.

Os resultados do presente estudo indicaram que existem diferenças significativas dos estados afetivos nos diferentes níveis de regularidade. Os fatores Vigor e Fadiga mostraram-se como os mais significativos nesta comparação. Dado o caracter pioneiro do presente estudo no que se refere a avaliar o impacto da regularidade de jogo nos estados afetivos não podemos fazer comparações com outros trabalhos. No entanto, os valores obtidos sugerem que quanto maior for envolvimento menor serão as manifestações de sintomas depressivos, de hostilidade e fadiga. Porém os sujeitos que mais jogam apresentam-se como mais vigorosos que os outros. .

Quanto às limitações do presente estudo, temos a dimensão da amostra, especialmente quando algumas das variáveis a estudar requerem que esta seja subdividida em grupos mais pequenos. Assim, para estudos futuros, recomendamos que se utilize a mesma técnica de amostragem (por conveniência), mas com o cuidado de a constituir por cachos em função das comparações a serem realizadas. Novos estudos deverão acautelar um número mínimo de participantes por grupo (15).

Um segundo aspeto a ser considerado é o tipo de variáveis a serem tidas em consideração. Sugerimos que replicas a este trabalho devem procurar avaliar parâmetros fisiológicos associados aos estados de humor/afetivos em avaliação, como por exemplo a condutividade da pele e a frequência cardíaca.

O fator Tensão (fator 1 da POMS) neste estudo mostrou efeitos estatísticos fortes e até diferenças significativas na presença/ ausência da realidade virtual. Seria por isso pertinente aprofundar o estudo sobre estes possíveis efeitos. Um outro estudo que gostaríamos de ver realizado seria uma investigação para perceber a partir de que momento um jogador assíduo/experiente começa a experienciar estados afetivos mais intensos e quais os fatores associados a este fenómeno.

\section{CONCLUSÕES}

O objetivo deste estudo foi comparar os estados afetivos dos participantes de duas 
condições/ambientes de realidade virtual (condição presença da realidade virtualutilização dos óculos de realidade virtual e condição ausência de realidade virtual-não utilização dos óculos de realidade virtual). Concluiu-se que existem diferenças dos estados afetivos nas duas condições bem como diferenças entre os sexos. Os fatores da POMS Tensão, Fadiga e Vigor são os mais significativos e os fatores Depressão, Hostilidade e Confusão apresentam um efeito estatístico nulo aquando das comparações.

\section{Agradecimentos:}

Os autores agradecem o apoio técnico e a facilidade em se utilizar as instalações do MASSIVE Lab. - INESC TEC Pólo UTẢD.

\section{Conflito de Interesses:}

Nada declarado.

\section{Financiamento:}

Nada declarado.

\section{REFERÊNCIAS}

Anderson, P., \& Price, M. (2007). The role of presence in virtual reality exposure therapy. Journal of Anxiety Disorders, 21, 742-751. Doi: 10.1016/j.janxdis.2006.11.002.

American Psychological Association (November 2017). Trends Report: what's ahead in 2018 for psychologists in technology, research, health care, advocacy, social justice \& more. Monitor in Psychology. DC; APA.

Arriaga, P., Silva, A., \& Esteves, F. (2001). Os efeitos de um jogo de computador nas aptidões perceptivas e espaciais. Psicologia: Teoria, Investigação e Prática, 269-284.

Baños, R. M., Botella, C., Alcañiz, M., Liaño, V., Guerrero, B., \& Rey, B. (2004). Immersion and emotion: Their impact on the sense of presence. CyberPsychology \& Behavior, 7(6), 734-741. Doi: 10.1089/cpb.2004.7.734.

Batista, M. D. L. S., Quintão, P. L., \& Barroso, S. M. (2008). Um Estudo sobre a Influência dos Jogos Eletrônicos sobre os Usuários. Revista
Eletrônica da Faculdade Metodista Granbery, 4, 2-11. Boot, W., Kramer, A., Simons, D., Fabiani, M., \& Gratton, G. (2008). The effects of video game playing on attention, memory, and executive control. Acta Psychologica 129, 387-398. Doi: 10.1016/j.actpsy.2008.09.005

Botella, C., Rivera, R. M. B., Palacios, A. G., Quero, S., Guillén, V., \& Marco, H. J. (2007). La utilización de las nuevas tecnologías de la información y la comunicación en psicología clínica. UOC Papers: revista sobre la societat del coneixement, (4), 32-41.

Carvalho, M. R., Freire, R. C., \& Nardi, A. E. (2008). Realidade virtual no tratamento do transtorno de pânico. J Bras Psiquiatric, 57(1), 64-69. Doi: 10.1590/S0047-20852008000100012.

Costa, R. M. E. M., \& de Carvalho, L. A. V. (2003, November). A Realidade Virtual como instrumento de inclusão social dos portadores de deficiências neuropsiquiátricas. In Brazilian Symposium on Computers in Education (Simpósio Brasileiro de Informática na Educação-SBIE) (Vol. 1, No. 1, pp. 405-414).

Dorval, M., \& Pervin, M. (1986). Effect of playing a video game on a measure of spatial visualization. Perceptual and Motor Skills, 62, 159-162. Doi: 10.2466/pms.1986.62.1.159.

Green, C. S., \& Bavelier, D. (2006). Effect of action video games on the spatial distribution of visuospatial attention. Journal of Experimental Psychology: Human Perception and Performance, 1465-1468. Doi: 10.1037/00961523.32.6.1465.

IJsselsteijn, W. A., \& Riva, G. (2003). Being there: The experience of presence in mediated environments. Being there: Concepts, effects and measurement of user presence in synthetic enviroments, 5.

Jaquet, C. (2013). A unidade do corpo e da mente: afetos, ações e paixões em Espinosa. Autêntica.

Kahneman, D. (1999). Objective happiness. In K. Daniel, Ed, D., \& Norbert, S., (3 ${ }^{\mathrm{a}}$ Ed.) Wellbeing: The foundations of hedonic psychology, (pp. 3-25). Estados Unidos da América.

Lopes, N., \& Oliveira, I. (2012). Videojogos e 
Desenvolvimento de Competências nos Estudantes Adultos. Atas do Encontro sobre Jogos e Mobile Learning, 35-45. Doi: http://hdl. handle.net/10400.2/2335.

Longhi, M. T., Bercht, M., \& Behar, P. A. (2007). Reconhecimento de estados afetivos do aluno em ambientes virtuais de aprendizagem. RENOTE, 5(2).

Mac Donald, R. (2006). Da Imersão à emoção: A presença em jogos digitais (Tese de Mestrado não publicada.) Universidade Vale do Itajaí.

Monteiro, L. M. C. (2011). Manipulação da emoção em ambientes de realidade virtual: Validação metodológica. (Tese de Doutoramento não publicada.) Universidade do Minho, Braga.

Netto, A. V., Machado, L. D. S., \& Oliveira, M. C. F. D. (2002). Realidade virtual-definições, dispositivos e aplicações. Revista Eletrônica de Iniciação Científica-REIC. Ano II, 2.

Okagaki, L., \& Frensch, P. A. (1994). Effects of video game playing on measures of spatial performance: Gender effects in late adolescence. Journal of Applied Developmental Psychology, 15, 33-58. Doi: 10.1016/01933973(94)90005-1.

Parsons, T. D., \& Rizzo, A. A. (2008). Affective outcomes of virtual reality exposure therapy for anxiety and specific phobias: A metaanalysis. Journal of behavior therapy and experimental psychiatry, 39(3), 250-261. Doi: 10.1016/j.jbtep.2007.07.007

Ribeiro, A., \& Monteiro, L. (2015). A indução afetiva em cenários de realidade virtual: avaliação da sensação de presença. Psicologia Clínica, 27(1), 139-160. Doi: 10.1590/010356652015000100008.

Ribeiro, M. W. S., \& Zorzal, E. R. (2011). Realidade virtual e aumentada: Aplicações e tendências. XIII Simpósio de Realidade Virtual e Aumentada, Uberlândia-MG-Brasil. Riva, G., Mantovani, F., Capideville, C. S.,
Preziosa, A., Morganti, F., Villani, D., ... Alcañiz, M. (2007). Affective interactions using virtual reality: The link between presence and emotions. CyberPsychology \& Behavior, 10(1), 45-56. Doi: 10.1089/ cpb.2006.9993.

Slater, M., Lotto, B., Arnold, M. M., \& Sanchez-Vives, M. V. (2009). How we experience immersive virtual environments: the concept of presence and its measurement. Anuario de psicología/The UB Journal of psychology, 40(2), 193-210.

Suzuki, F. T. I., Matias, M. V., Silva, M. T. A., \& Oliveira, M. P. M. T. D. (2009). O uso de videogames, jogos de computador e internet por uma amostra de universitários da Universidade de São Paulo. Jornal Brasileiro de Psiquiatria, 58(3), 162-168. Doi: http:// producao.usp.br/handle/BDPI/12049.

Strickland, D. (1997). Virtual reality for the treatment of autism. Virtual Reality in NeuroPsycho-Physiology. 81-86.

Tijiboy, A. V., Pereira, E. A., \& Woiciechoski, L. R. (2009). Interação com Afeto: Aprendizagem em Ambientes Virtuais de Aprendizagem. RENOTE, 7(1).

Viana, M., Almeida, P., \& Santos, R. (2001). Adaptação portuguesa da versão redizida do perfil de estados de humor: POMS. Análise Psicológica, 19(1), 77-92.

Vasconcelos-Raposo, J., Bessa, M., Melo, M., barbosa, L., Rodrigues, R., Teixeira, C., ... \& Sousa, A. (2016). Adaptation and validation of the Igroup Presence Questionnaire (IPQ) in a portuguese sample. PRESENCE: Teleoperators and Virtual Environments, 25(3), 1-13. Doi: 10.1162/PRES_a_00261

Yannakakis, G. N., Martinez, H. P., \& Garbarino, M. (2016). Psychophysiology in games. In Emotion in Games, (pp.119-137). Springer International Publishing. Doi: 10.1007/9783-319-41316-7 7. 